Chitra Tirunal Institute of Medical Sciences and Technology, Trivandrum, India; ${ }^{7}$ Madras Diabetes Research Foundation, Chennai, India; ${ }^{8}$ Indira Gandhi Government Medical College, Nagpur, India

Introduction Cardiovascular disease (CVD) prevention guidelines recommend lifetime risk stratification for primary prevention of CVD, but no such risk stratification has been performed in India to date.

Methods We estimated short-term and lifetime predicted CVD risk among 10054 disease free, adult Indians in the age group of 20-69 years who participated in a nationwide risk factor surveillance study. The study population was then stratified into high shortterm ( $\geq 10 \% 10$-year risk or diabetes), low short-term $(<10 \%) /$ high lifetime and low short-term/low lifetime CVD risk groups.

Results The mean age (SD) of the study population (men=63\%) was $40.8+10.9$ years. High short-term risk for coronary heart disease was prevalent in more than one fifth of the population $(23.5 \%, 95 \%$ CI 22.7 to 24.4). Nearly half of individuals with low short-term predicted risk $(48.2 \%, 95 \%$ CI 47.1 to 49.3$)$ had a high predicted lifetime risk for CVD. While the proportion of individuals with all optimal risk factors was $15.3 \%$ (95\% CI 14.6 to 16.0 ), it was $20.6 \%$ (95\% CI 18.7 to 22.6 ) and $8.8 \%$ (95\% CI 7.7 to 10.5$)$ in the highest and lowest educational groups, respectively.

Conclusion Approximately 1 in 2 men and 3 in 4 women in India had low short-term predicted risks for CVD in this national study, based on aggregate risk factor burden. However, 2 in 3 men and 1 in 2 women had high lifetime predicted risks for CVD, highlighting a key limitation of short-term risk stratification.

\section{P2-129 PRE-PREGNANCY BODY MASS INDEX AND BLOOD PRESSURE IN THE FIRST TRIMESTER OF PREGNANCY: PRELIMINARY RESULTS FROM A COHORT OF RIO DE JANEIRO, BRAZIL}

\section{doi:10.1136/jech.2011.142976i.64}

F Rebelo, A B Franco-Sena, D R Farias, J dos Santos Vaz, G Kac.* Rio de Janeiro Federal University, Rio de Janeiro, Brazil

Background Women who have higher systolic blood pressure (SBP) and diastolic blood pressure (DBP) in early pregnancy are more prone to develop pregnancy complications such as hypertension, preeclampsia and eclampsia. These complications contribute significantly to morbidity, stillbirth and neonatal mortality.

Objective To evaluate the effect of pre-pregnancy body mass index (BMI) on first trimester SBP, DBP and mean arterial pressure (MAP). Methods 118 pregnant women between 20 and 40 years of age, up to 13 weeks and free from chronic or infectious diseases were investigated. SBP and DBP were obtained with automatic arm sphygmomanometer (Omron) and MAP was calculated as (SBP $+\mathrm{DBP} \times 2) / 3$. Statistical analysis was performed using ANOVA, correlation and multivariate linear regression. Adjustments were made for age, skin colour, parity and smoking.

Results Women were 26.3 44.9 years of age, had $8.7 \pm 2.7$ years of schooling, per-capita income (US\$) of $291.2 \pm 166.4$ and pre-pregnancy BMI of $25.6 \pm 5.1 \mathrm{~kg} / \mathrm{m}^{2}$. There was a moderate positive correlation between pre-pregnancy BMI and SBP $(r=0.52 ; p<0.001)$, $\operatorname{DBP}(r=0.33 ; \mathrm{p}<0.001)$ and MAP $(\mathrm{r}=0.44 ; \mathrm{p}<0.001)$. Mean MAP $(\mathrm{mm} \mathrm{Hg})$ increased with BMI categories (underweight $=76.5$; normal weight $=77.4$; overweight $=82.9$ and obesity $=88.7, p<0.001$ ) as did SBP (underweight $=100.3$; normal weight $=105.8$; overweight $=112.4$ and obesity $=121.2, \quad \mathrm{p}<0.001$ ) and DBP (underweight $=64.8 ;$ normal weight $=63.5 ; \quad$ overweight $=68.3$ and obesity $=72.6, \mathrm{p}<0.001)$. Multivariate linear regression showed that an increase of $1.0 \mathrm{~kg} / \mathrm{m}^{2}$ in pre-pregnancy BMI raised SBP in $1.24 \mathrm{~mm} \mathrm{Hg}(\mathrm{p}<0.001)$ and $0.53 \mathrm{~mm} \mathrm{Hg}$ in DBP $(p=0.002)$.
Conclusion Women who begin pregnancy overweight or obese tend to have higher values of SBP and DBP, which may indicate a higher risk of developing hypertensive disorders during pregnancy.

\section{P2-130 EPIDEMIOLOGY OF HYPERTENSION IN AN URBAN SRI LANKAN POPULATION}

doi:10.1136/jech.2011.142976i.65

${ }^{1} \mathrm{~A}$ Kasturiratne, ${ }^{*}{ }^{1} \mathrm{~T}$ Warnakulasuriya, ${ }^{1} \mathrm{~J}$ Pinidiyapathirage, ${ }^{2} \mathrm{~N}$ Kato, ${ }^{1} \mathrm{R}$ Wickremasinghe, ${ }^{1} \mathrm{~A}$ Pathmeswaran. ${ }^{1}$ Department of Public Health, Faculty of Medicine, University of Kelaniya, Ragama, Sri Lanka; ${ }^{2}$ Department of Gene Diagnostics and Therapeutics, Faculty of Medicine, University of Kelaniya, Tokyo, Japan

Introduction Hypertension is a common risk factor for cardiovascular disease. In Sri Lanka, despite the existence of a universal free health system, services are not available for routine screening of hypertension in the general population. This paper aims to describe the epidemiology of hypertension in 35-64 year old residents in Ragama Medical Officer of Health area in the Gampaha district, Sri Lanka.

Methods An age-stratified random sample of 4400 adults between 35 and 64 years of age drawn from the population based electoral list, was invited for a screening programme on cardiovascular risk factors. Socio-demographic and risk factor related data and anthropometric and blood pressure measurements were obtained by trained research assistants. Blood was obtained for relevant biochemical investigations.

Results The prevalence of hypertension (systolic $>139 \mathrm{~mm} \mathrm{Hg}$ and/or diastolic $>89 \mathrm{~mm} \mathrm{Hg}$ ) in 2986 subjects (Males 45\%), was $30.4 \%$ (27.8\% in males; $32.5 \%$ in females). $31.8 \%(n=288)$ were previously undetected. Of the known hypertensives, $19.5 \%$ were not on anti-hypertensive medication and only $32.1 \%$ were controlled (defined by systolic <140 $\mathrm{mm} \mathrm{Hg}$ and diastolic <90 mm Hg). Factors associated with hypertension in both males and females were body mass index, waist circumference, fasting blood glucose and serum triglycerides.

Conclusions The prevalence observed is comparable to the prevalences of developed countries with relatively older populations. A considerable proportion of known hypertensives are not on treatment and the observed poor control indicates problems in drug compliance. Interventions targeting lifestyle modification and drug compliance are essential to control adverse outcomes of hypertension.

\section{P2-131 FALLING PREVALENCE OF IMPAIRED GLUCOSE TOLERANCE IN SOUTH ASIAN POPULATIONS}

doi:10.1136/jech.2011.142976i.66

${ }^{1} \mathrm{~S}$ V Katikireddi, ${ }^{2} \mathrm{~J}$ Morling, ${ }^{2} \mathrm{R}$ Bhopal. ${ }^{1} \mathrm{NHS}$ Lothian, Edinburgh, Scotland, UK ${ }^{2}$ Centre for Population Health Sciences, University of Edinburgh, Edinburgh, Scotland, UK

Introduction Recently, diabetes prevalence has soared in South Asians making it a global public health priority. However, there are suggestions from the PODOSA trial and elsewhere that pre-diabetes, including impaired glucose tolerance (IGT), may not be increasing. We conducted a systematic review to assess secular trends in prediabetes in South Asian populations worldwide.

Methods We searched electronic databases from inception to June 2009 for cross-sectional studies providing prevalence of pre-diabetes (using WHO criteria) in South Asian adult populations. Two reviewers independently screened articles, performed data extraction, quality appraisal and study classification with any discrepancies resolved by consensus. Repeated cross-sectional studies, 